\title{
Combinatorial algorithms for inverse absolute and vertex 1-center location problems on trees
}

\author{
Behrooz Alizadeh*† Rainer E. Burkard *
}

August 13, 2009, revised June 18, 2010

\begin{abstract}
In an inverse network absolute (or vertex) 1-center location problem the parameters of a given network, like edge lengths or vertex weights, have to be modified at minimum total cost such that a prespecified vertex $s$ becomes an absolute (or a vertex) 1-center of the network. In this article, the inverse absolute and vertex 1-center location problems on unweighted trees with $n+1$ vertices are considered where the edge lengths can be changed within certain bounds. For solving these problems a fast method is developed for reducing the height of one tree and increasing the height of a second tree under minimum cost until the heights of both trees become equal. Using this result a combinatorial $O\left(n^{2}\right)$ time algorithm is stated for the inverse absolute 1-center location problem in which no topology change occurs. If topology changes are allowed, an $O\left(n^{2} r\right)$ time algorithm solves the problem where $r, r<n$, is the compressed depth of the tree network $T$ rooted in $s$. Finally, the inverse vertex 1-center problem with edge length modifications is solved on $T$. If all edge lengths remain positive this problem can be solved within the improved $O\left(n^{2}\right)$ time complexity by balancing the height of two trees. In the general case one gets the improved $O\left(n^{2} r_{v}\right)$ time complexity where the parameter $r_{v}$ is bounded by $n$.
\end{abstract}

Keywords: network center location, inverse optimization, combinatorial optimization, design of algorithms

\section{Introduction}

Location problems are fundamental optimization models in operations research and play a considerable role in practice and theory. These problems are concerned with determining the optimal locations of one or more new facilities in network systems or in space in order

\footnotetext{
*Institute of Optimization and Discrete Mathematics, Graz University of Technology, Steyrergasse 30, A-8010 Graz, Austria. \{alizadeh, burkard\}@opt.math.tugraz.at

${ }^{\dagger}$ Department of Applied Mathematics, Faculty of Basic Sciences for Engineering, Sahand University of Technology, Tabriz, Iran. alizadeh@sut.ac.ir
}

Corresponding author: Rainer E. Burkard

The first author acknowledges financial support by the NAWI-Project under the grant F-NW-MATH-05. This research has also been supported by the Austrian Science Fund (FWF) Project P18918-N18. 
to fulfill the demands of customers. See the books of Daskin [6], Drezner and Hamacher [7], Francis, McGinnis and White [8], Love, Morris and Wesolowsky [18], and Mirchandani and Francis [21] for detailed surveys on location problems.

The 1-center location problem, or simply center location problem, is the special case where one center is to be located such that the maximum weighted distance to the given points becomes minimum. Such problems occur when the best location of an emergency service, a hospital, a fire station, a police office, a bank branch, a train station, an airport, a shopping center, a city park or another facility center has to be found. One of the most important center location models is the classical network 1-center location problem which is stated in the following way. Let a connected graph $G=(V(G), E(G))$ with vertex set $V(G),|V(G)|=n$, and edge set $E(G),|E(G)|=m$, be given. Every edge $e \in E(G)$ has a positive length $\ell(e)$. The shortest path distance between two vertices $u$ and $v$ is denoted by $d_{\ell}(u, v)$. Moreover, for any vertex $v \in V(G)$, let $w(v)$ be a nonnegative vertex weight. We say that point $p$ lies in $G, p \in G$, if $p$ coincides with a vertex or lies on an edge $e=u v$ of $G$. In the latter case $p$ is fixed by choosing a parameter $\lambda, 0<\lambda<1$, such that $d_{\ell}(u, p)=\lambda \ell(e)$. In the classical network 1-center location problem, the task is to find a point $p \in G$ such that the maximum weighted distance from any vertex $v \in V(G)$ to point $p$ becomes minimum, or,

$$
\begin{array}{ll}
\text { minimize } & \max _{v \in V(G)} w(v) d_{\ell}(v, p) \\
\text { subject to } & p \in G .
\end{array}
$$

A point $p^{*}$ which solves problem (1), is said to be an absolute 1-center location. If point $p$ in problem (1) is restricted to be a vertex (i.e., $p \in V(G)$ ), then we say that the optimal solution $p^{*}$ is a vertex 1 -center location of $G$.

Kariv and Hakimi [16] designed an $O(m n \log n)$ algorithm for finding an absolute 1center of a weighted network and an $O\left(m n+n^{2} \log n\right)$ algorithm for finding an absolute 1-center of an unweighted network, provided that the distance matrix of the network is available. Moreover, they proposed an $O(n \log n)$ time algorithm for the absolute (or vertex) 1-center location problem on weighted trees. Later, in 1983, Megiddo [20] showed that the weighted 1-center of a tree can be obtained in $O(n)$ time, since the objective function of the problem is convex on every simple path of the tree. For the unweighted case an efficient $O(n)$ time algorithm was developed by Handler [12].

Inverse optimization problems, particularly inverse location problems have found a significant interest in recent years. Since we are usually confronted with the situation that facilities already exist and cannot be relocated, inverse location problems play an important role in practice. Given a feasible solution for a location problem, the inverse location problem is concerned with modifying parameters of the original problem at minimum total cost within certain modification bounds such that the given feasible solution becomes an optimal solution with respect to the new parameter values. For example, consider the train station of a city which can not be relocated. The mayor could change some parameters in the urban system (e.g., improving streets or urban transportation lines) at minmimum cost subject to evident length constraints such that the current location of the train station becomes the center (in a graph theoretic sense) of the city.

A detailed survey on inverse optimization problems has been compiled by Heuberger [14]. In the context of location problems Cai, Yang and Zhang [5] proved that the inverse 
1-center location problem with edge length modification on general unweighted directed graphs is $\mathcal{N} \mathcal{P}$-hard, while the underlying center location problem is solvable in polynomial time. In 2004, Burkard, Pleschiutschnig and Zhang [3] considered inverse $p$-median problems and showed that discrete inverse $p$-median location problems can be solved in polynomial time, when $p$ is fixed and not an input parameter. They proposed a greedy-like $O(n \log n)$ time algorithm for the inverse 1-median problem with vertex weight modifications on tree networks. Hatzl [13] as well as Galavii [9] showed later that this problem can actually be solved in $O(n)$ time. Moreover, Burkard et al. [3] proved that the inverse 1-median problem on the plane under Manhattan (or Chebyshev) norm can be solved in $O(n \log n)$ time. Later the same authors [4] investigated the inverse 1-median problem with vertex weight modification and unit cost on a cycle. They showed that this problem can be solved in $O\left(n^{2}\right)$ time by using methods from computational geometry. In 2007, Gassner [10] suggested an efficient $O(n \log n)$ time solution method for the inverse 1-maxian problem with edge length modifications on tree networks. The inverse FermatWeber problem was studied by Burkard, Galavii and Gassner [2]. The authors derived a combinatorial approach which solves the problem in $O(n \log n)$ time for unit cost and under the assumption that the prespecified point that should become a 1-median does not coincide with a given point in the plane. Galavii [9] showed in his Ph.D. thesis that the 1-median on a path with pos/neg weights lies in one of the vertices with positive weights or lies in one of the end points of the path. This property allows the inverse 1-median problem to be solved on a path with negative weights in $O(n)$ time. Gassner [11] considered an inverse version of the convex ordered median problem and showed that this problem is $\mathcal{N} \mathcal{P}$-hard on general graphs, even on trees. Further, it was shown that the problem remains $\mathcal{N} \mathcal{P}$-hard for unit weights or if the underlying problem is a $k$-centrum problem (but not, if both of these conditions hold). The inverse unit-weight $k$-centrum problem with unit cost coefficients on a tree can be solved in $O\left(n^{3} k^{2}\right)$ time. Recently, Yang and Zhang [23] proposed an $O\left(n^{2} \log n\right)$ time solution method for the inverse vertex center problem on an unweighted tree provided that the modified edge lengths always remain positive. Dropping this condition, they mention that the general problem can be solved in $O\left(n^{3} \log n\right)$ time.

This article develops novel combinatorial solution methods for the inverse absolute (or vertex) 1-center location problem where the edge lengths of a given unweighted tree network are modified at minimum total cost with respect to modification bounds such that a prespecified vertex $s$ becomes an absolute (or a vertex) 1-center. The article is organized as follows: In the next section, we state the inverse absolute (or vertex) 1center location problem on unweighted tree networks and show that the problem can be formulated as a nonlinear semi-infinite (or nonlinear) optimization model. By recalling basic properties from the classical absolute and vertex 1-center location problem, we discuss the main ideas of purely combinatorial solution algorithms. In Section 3 the tree height reduction problem is considered where the height of a given rooted tree is to be reduced at minimum cost by a prespecified amount. The solution method for this problem is applied in Section 4 to develop an exact algorithm for balancing the heights of two rooted trees by increasing the height of one tree and decreasing the height of the second tree under minimum cost. The latter algorithm is used in Subsection 5.1 to solve the inverse absolute 1-center location problem in $O\left(n^{2}\right)$ time where we assume that no 
topology change is admitted. Dropping this condition, we develop an exact algorithm for the general case with $O\left(n^{2} r\right)$ time complexity in Subsection 5.2 where $r, r<n$, is the compressed depth of the given tree rooted in the vertex $s$. Finally, in Section 6 , the height balancing algorithm is again used for solving the inverse vertex 1-center location problem on an unweigted tree. It yields improved time complexities for both cases, when the modified edge lengths remain positive and the general case.

\section{Problem statement and main solution ideas}

\subsection{Problem formulation}

Let an unweighted tree network $T=(V(T), E(T))$ with vertex set $V(T),|V(T)|=n+1$, and edge set $E(T)$ be given such that every edge $e \in E(T)$ has a positive length $\ell(e)$. Let $s$ be a prespecified vertex of $T$. We want to modify the edge lengths at minimum total cost such that $s$ becomes the absolute (or vertex) 1-center. Suppose that we incur nonnegative cost $c^{+}(e)$, if $\ell(e)$ is increased by one unit and we incur nonnegative cost $c^{-}(e)$, if $\ell(e)$ is reduced by one unit. Moreover, we assume that it is not possible to increase and reduce the edge lengths arbitrarily. Namely, every edge length $\ell(e)$ can only be changed between a lower bound $\ell_{\text {low }}(e) \geq 0$ and an upper bound $\ell_{\text {upp }}(e)$. Therefore, we can state the inverse absolute (or vertex) 1-center location problem on the given tree network $T$ as follows: hold:

Modify the edge lengths $\ell(e), e \in E(T)$, to $\tilde{\ell}(e)$ such that the following three statements

(i) The vertex $s$ becomes an absolute (or a vertex) 1-center of $T$ with respect to $\tilde{\ell}$, i.e., for all $p \in T$ (or $p \in V(T)$ ),

$$
\max _{v \in V(T)} d_{\tilde{\ell}}(v, s) \leq \max _{v \in V(T)} d_{\tilde{\ell}}(v, p) .
$$

(ii) The linear cost function

$$
\sum_{e \in E(T)}\left(c^{+}(e) x(e)+c^{-}(e) y(e)\right)
$$

becomes minimum, where $x(e)$ and $y(e)$ are the amounts by which the edge length $\ell(e)$ is increased and reduced, respectively.

(iii) The new edge lengths lie within given modification bounds

$$
\ell_{\text {low }}(e) \leq \tilde{\ell}(e) \leq \ell_{\text {upp }}(e) \quad \text { for all } e \in E(T) .
$$

Hence, based on the problem statement mentioned above, the inverse absolute (or vertex) 1-center location problem on the tree network $T$ can be formulated as the following nonlinear semi-infinite (or nonlinear) optimization model: 


$$
\begin{aligned}
\min _{x, y} & \sum_{e \in E(T)}\left(c^{+}(e) x(e)+c^{-}(e) y(e)\right) \\
\text { subject to } & \max _{v \in V(T)} d_{\tilde{\ell}}(v, s) \leq \max _{v \in V(T)} d_{\tilde{\ell}}(v, p) \quad \text { for all } p \in T(\text { or } p \in V(T)), \\
& \tilde{\ell}(e)=\ell(e)+x(e)-y(e) \quad \text { for all } e \in E(T), \\
& x(e) \leq \ell^{+}(e) \quad \text { for all } e \in E(T), \\
& y(e) \leq \ell^{-}(e) \quad \text { for all } e \in E(T), \\
& x(e), y(e) \geq 0 \quad \text { for all } e \in E(T),
\end{aligned}
$$

where $\ell^{+}(e)=\ell_{\text {upp }}(e)-\ell(e)$ and $\ell^{-}(e)=\ell(e)-\ell_{\text {low }}(e)$ are the maximum feasible amounts by which $\ell(e)$ can be increased and reduced, respectively. Every feasible solution vector $(x, y)=(x(e), y(e))_{e \in E(T)}$ is also called a feasible modification of the inverse absolute (or vertex) 1-center location problem.

\subsection{Main ideas based on optimality criteria for the classical center prob- lems}

The solution methods for the inverse 1-center problems under investigation are based on optimality criteria for clssical 1-center problems. Handler [12] showed:

Theorem 2.1 (midpoint-property)

In an unweighted tree network the midpoint of a longest path is an absolute 1-center. The closest vertex to the absolute 1-center is a vertex 1-center of the given network.

Moreover, Handler [12] proved the following Lemma:

Lemma 2.2 The absolute 1-center of an unweighted tree network is unique.

Lemma 2.2 means that the unique absolute 1-center of an unweighted tree is the midpoint of all longest paths. Therefore, the main idea for solving the inverse absolute 1-center location problem on a tree network $T$ is the following: the given tree $T$ is split into two subtrees $L$ and $R$ where $L$ is given by a longest path from $s$ to a leaf together with all its adjacent vertices, whereas the subtree $R$ rooted in $s$ contains all other vertices (see Fig.1). If the heights of $L$ and $R$ are not equal, then the length of some edges in $L$ has to be reduced and the length of some edges in $R$ has to be increased. The edge lengths are modified at minimum total cost with respect to the given modification bounds until the prespecified vertex $s$ fulfills the midpoint-property on $T$.

The splitting of $T$ into two subtrees $L$ and $R$ is done in the following way. Let $\operatorname{deg}(s)$ be the degree of the prespecified vertex $s$. If $\operatorname{deg}(s)=1$, then we set

$$
L=T, \quad R=s .
$$

Otherwise we partition $T$ into nontrivial subtrees $T_{1}, T_{2}, \ldots, T_{\operatorname{deg}(s)}$ such that

$$
\bigcup_{i=1}^{\operatorname{deg}(s)} T_{i}=T \quad \text { and } \quad T_{i} \cap T_{j}=s \quad \text { for } 1 \leq i, j \leq \operatorname{deg}(s), i \neq j .
$$


Let $P_{u v}$ denote the unique path between two arbitrary vertices $u, v \in V(T)$ and let $\ell\left(P_{u v}\right)$ be the length of $P_{u v}$ with respect to $\ell$. Without loss of generality a longest path $P_{s z^{*}}$ from $s$ to the leaves of $T$ is contained in $T_{1}$. Then we define

$$
L=T_{1} \quad, \quad R=\bigcup_{i=2}^{\operatorname{deg}(s)} T_{i} .
$$

Figure 1 illustrates the partitioning of the tree $T$ into subtrees $L$ and $R$ for the case $\operatorname{deg}(s) \geq 2$.

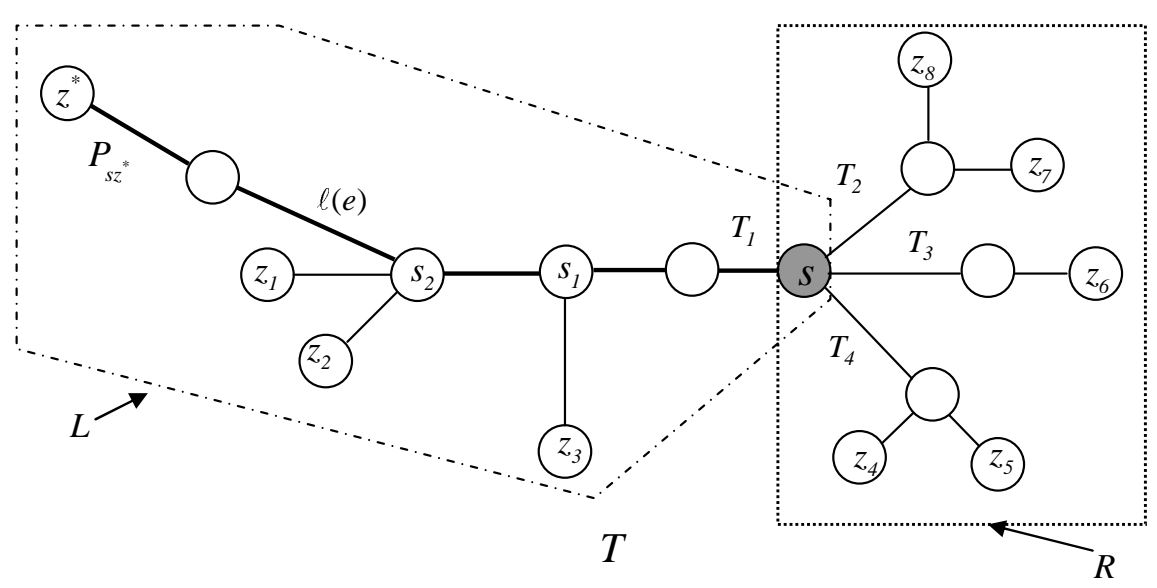

Figure 1: Illustration for partitioning the given tree network $T$ into subtrees $L$ and $R$ where $\operatorname{deg}(s) \geq 2$. The path $P_{s z^{*}}$ is a longest path from $s$ to the leaves of $T$

If subtree $L$ is not a path let $s_{1}$ denote the nearest vertex to $s$ in the subtree $L$ with $\operatorname{deg}\left(s_{1}\right) \geq 3$. We say that an essential topology change occurs if the length $\ell\left(P_{s s_{1}}\right)$ is reduced to zero, i.e., if vertex $s_{1}$ coincides with vertex $s$ in the reduced tree. In this case some vertices of $L$ move to the right tree $R$. Thus we get new left and right trees. For example, consider the tree $T$ given in Figure 1. After reducing the edge length $\ell\left(P_{s s_{1}}\right)$ to 0 , the leaf $z_{3}$ moves in the reduced tree $T^{1}$ from the left tree $L$ to the right tree $R^{1}$, see Figure 2.

Note that an essential topology change can only occur if $\ell^{-}(e)=\ell(e)$ for all $e \in$ $E\left(P_{s s_{1}}\right)$. Moreover, observe that an essential topology change never occurs if $L$ is a path.

Now let us turn to the inverse vertex 1-center location problem. Our solution approach for this problem relies on the following ideas:

Let $Z(T)$ denote the set of leaves of $T$. Consider a longest path $P_{s z^{*}}, z^{*} \in Z(T)$, from the prespecified vertex $s$ to the leaves. Let $a(s)$ be the unique adjacent vertex to $s$ on this path $P_{s z^{*}}$ and let $e_{s}$ be the unique edge with end points $s$ and $a(s)$. If we delete edge $e_{s}$, then the tree network $T$ is split into two disjoint subtrees $\hat{L}$ and $\hat{R}$ such that

$$
\hat{L} \cup \hat{R}=T-e_{s} \quad, \quad \hat{L} \cap \hat{R}=\emptyset,
$$




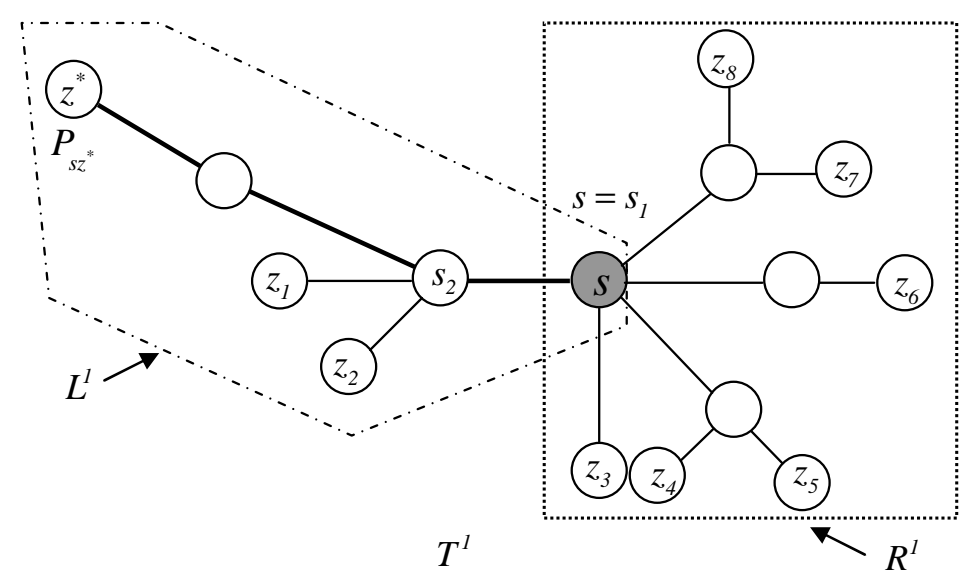

Figure 2: Tree $T^{1}$ obtained from tree $T$ in Figure 1 by reducing the length of path $P_{s s_{1}}$ to 0 . The leaf $z_{3}$ which lies in $T$ in the left tree $L$ moves after the reduction to the right tree $R^{1}$ of $T^{1}$.

and

$$
a(s) \in V(\hat{L}) \quad, \quad s \in V(\hat{R}) .
$$

We root subtree $\hat{L}$ in vertex $a(s)$ and subtree $\hat{R}$ in vertex $s$. The proposed solution algorithms for solving the inverse vertex 1-center location problem are based on the following lemma (see also Yang and Zhang [23]):

Lemma 2.3 Given an unweighted tree network $T$, a vertex $s$ is a vertex 1-center location if and only if the inequality

$$
\ell\left(P_{s z^{*}}\right) \leq \max \left\{\ell\left(P_{a(s) z}\right): z \in Z(\hat{R})\right\}
$$

holds where $Z(\hat{R})$ is the set of leaves of the rooted subtree $\hat{R}$.

Proof. According to Theorem 2.1 the closest vertex to the absolute 1-center is a vertex 1-center of the unweighted tree. This immediately yields this lemma.

Lemma 2.3 leads to the following main idea for solving the inverse vertex 1-center problem: split $T$ into two rooted subtrees $\hat{L}$ and $\hat{R}$. If the optimality inequality (7) is not satisfied, then reduce the edge lengths on $\hat{L}$ and increase the edge lengths on $\hat{R}$ at minimum total cost with respect to the given modification bounds such that the maximum distance from $a(s)$ to the leaves $z \in Z(\hat{L})$ becomes equal to the maximum distance from $s$ to the leaves $z \in Z(\hat{R})$.

In the next section, we discuss the problem of reducing the height of a tree. In Sections 5 and 6 we apply this to the subtrees $L$ and $\hat{L}$, respectively. 


\section{Tree height reduction problem}

Let $T=(V(T), E(T))$ be a tree with vertex set $V(T)$ and edge set $E(T)$ which is rooted in a vertex $s$. Every edge $e \in E(T)$ has a positive length $\ell(e)$. Reducing this length incurs nonnegative cost $c(e)$ per unit. Furthermore, let $h_{\ell}(T)$ denote the height of $T$ which is equal to the length of a longest path from the root to the leaves of $T$ with respect to the edge lengths $\ell$. The tree height reduction problem is concerned with reducing $h_{\ell}(T)$ by a given amount $\delta h$, the so-called reduction argument, at minimum total cost. Suppose that $\ell^{-}(e)$ is the maximum feasible amount by which the length $\ell(e)$ can be reduced. In the tree height reduction problem on $T$, the goal is to reduce the edge lengths $\ell(e)$ by amounts $y(e)$ with respect to the given bounds $0 \leq y(e) \leq \ell^{-}(e)$ for all $e \in E(T)$ such that the total cost

$$
\sum_{e \in E(T)} c(e) y(e)
$$

becomes minimum while the height of $T$ is reduced by the amount $\delta h$.

In the following we are going to present a solution method for the tree height reduction problem which is similar to an idea of Zhang, Liu and Ma [24] developed for solving reverse center location problems. The method relies on a sequence of minimum $s-t$ cuts in an auxiliary tree-like network $N$ defined in the following way. Given the rooted tree $T$, we introduce a new vertex $t$ and connect it to every leaf $z \in Z(T)$. Then we define

$$
V(N)=V(T) \cup\{t\} \text { and } E(N)=E(T) \cup\{e=z t: z \in Z(T)\} .
$$

Moreover, let

$$
\begin{aligned}
& \ell_{N}(e)= \begin{cases}\ell^{-}(e) & \text { if } e \in E(T), \\
h_{\ell}(T)-d_{\ell}(s, z) & \text { if } e=z t, z \in Z(T),\end{cases} \\
& c_{N}(e)= \begin{cases}c(e) & \text { if } e \in E(T), \ell_{N}(e)>0, \\
0 & \text { if } e=z t, z \in Z(T), \ell_{N}(e)>0, \\
M & \text { otherwise, }\end{cases}
\end{aligned}
$$

where $M$ is a very big value. The network $N=\left(V(N), E(N), \ell_{N}, c_{N}\right)$ is called the auxiliary tree-like network of $T$ with vertex set $V(N)$, edge set $E(N)$ and edge lengths $\ell_{N}$. All edges are directed from $s$ to $t$ and have capacities $c_{N}(e), e \in E(N)$ (see Figure 3). Furthermore, define the paths

$$
P_{z}=P_{s z} \cup\{z t\} \quad \text { for all } z \in Z(T),
$$

on network $N$ where $P_{s z}$ is the unique path from $s$ to the leaf $z$ on tree $T$. Thus we get the following lemma:

Lemma 3.1 There exists a one-to-one correspondence between feasible solutions of the following two problems (a) and (b) with same objective function values:

Problem (a): reduce the height $h_{\ell}(T)$ of tree $T$ by the amount $\delta h$

Problem (b): reduce all path lengths $\ell_{N}\left(P_{z}\right), z \in Z(T)$, by the amount $\delta h$ 


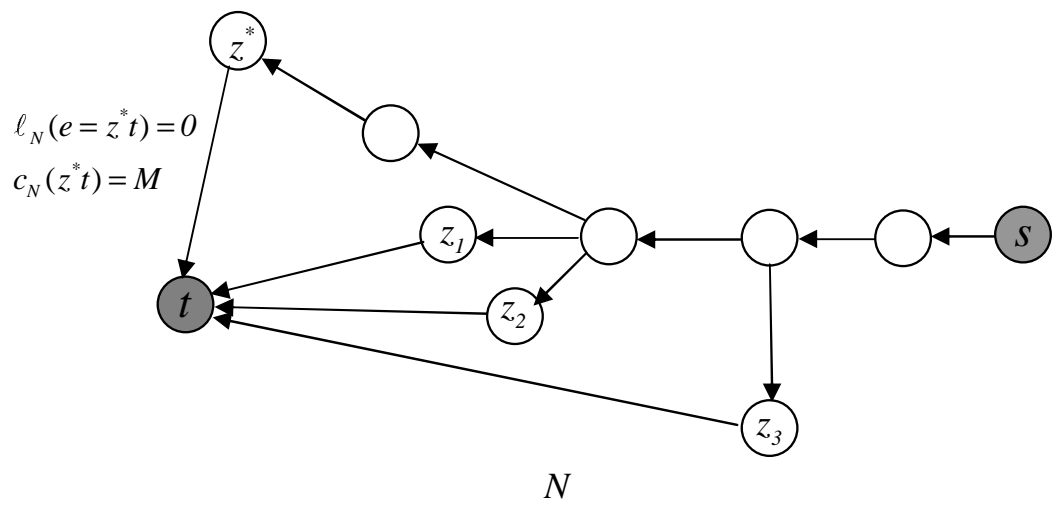

Figure 3: $N$ is the auxiliary tree-like network of the subtree $L$ shown in Figure 1.

Now we show that by finding a minimum $s-t$ cut on network $N$ one can reduce all path lengths $\ell_{N}\left(P_{z}\right), z \in Z(T)$, and consequently according to Lemma 3.1, the height of the given tree $T$ at minimum cost by changing the lengths of those edges which are contained in the minimum $s-t$ cut of $N$. Note that in network $N$ we are allowed to reduce the length of every edge to zero.

Let $K=(X, Y)$ with $s \in X, t \in Y$ be a minimum $s-t$ cut in $N$ and $E(K)=\{e=$ $u v \in E(N): u \in X, v \in Y\}$. The capacity of $K$ is computed by

$$
c(K)=\sum_{e \in E(K)} c_{N}(e) .
$$

If $c(K) \geq M$, then there exists at least one path $P_{z}, z \in Z(T)$, with $\ell_{N}(e)=0$ for all $e \in E\left(P_{z}\right)$. In this case the length of $P_{z}$ cannot be reduced any more and thus the tree height reduction problem is infeasible. Otherwise, let

$$
\delta(K)=\min \left\{\ell_{N}(e): e \in E(K)\right\} .
$$

The parameter $\delta(K)$ is the maximum amount by which the length of all edges in $E(K)$ can be reduced. The following proposition is crucial for the correctness of the solution method for the tree height reduction problem.

Proposition 3.2 Let $K$ be a minimum $s-t$ cut in $N$ with $c(K)<M$ and let $0<\delta \leq$ $\delta(K)$. If we reduce the edge lengths of $N$ by amounts

$$
y^{*}(e)= \begin{cases}\delta & \text { if } e \in E(K), \\ 0 & \text { otherwise },\end{cases}
$$

then the height of tree $T$ is reduced by the amount $\delta$ at minimum total cost.

Proof. Let $K$ be a minimum $s-t$ cut with $c(K)<M$. This implies that all edges $e \in E(K)$ have a positive length. Therefore $\delta(K)>0$. The cut contains in particular 
an edge of every longest path $P_{s z}$ in $T$, since $c_{N}(z t)=M$. Therefore all longest paths in $T$ are reduced by $\delta$. If $P_{s z}$ is not a longest path in $T$, the cut contains the edge $z t$, since $c_{N}(z t)=0$ and the cut has minimum cost. The definition of $\delta(K)$ implies that after the reduction all reduced longest paths have a length at least as long as a second longest path $P_{s z}$ in $T$ before the reduction. Thus the height of $T$ has been reduced by $\delta$ at minimum cost.

Let us now assume that the height of tree $T$ has to be reduced by the amount $\delta h$. We determine a first minimum $s-t$ cut $K_{1}$ in network $N$ with corresponding value $\delta\left(K_{1}\right)$. If $\delta h \leq \delta\left(K_{1}\right)$, the height can be reduced by using the cut $K_{1}$. Otherwise, choose in the first step $\delta=\delta\left(K_{1}\right)$, reduce the edge lengths in $N$ by

$$
\ell_{N}(e)= \begin{cases}\ell_{N}(e)-\delta\left(K_{1}\right) & \text { if } e \in E\left(K_{1}\right), \\ \ell_{N}(e) & \text { otherwise }\end{cases}
$$

and then update

$$
\begin{gathered}
\delta h=\delta h-\delta\left(K_{1}\right), \\
c_{N}(e)= \begin{cases}c_{N}(e) & \text { if } \ell_{N}(e)>0, \\
M & \text { otherwise. }\end{cases}
\end{gathered}
$$

The resulting network is again called $N=\left(V(N), E(N), \ell_{N}, c_{N}\right)$. Observe that after such a transformation at least one edge $e \in E(N)$ will have the new length $\ell_{N}(e)=0$. Hence, by iterating this process we find a sequence of minimum $s-t$ cuts $K_{i}, i=1, \ldots q$, with capacities $c\left(K_{i}\right)$ which lead successively to the reduction of the underlying tree $T$ by the amounts $\delta\left(K_{i}\right), i=1, \ldots q$. The process terminates if either $\delta\left(K_{q}\right) \geq \delta h$ or if $C\left(K_{q}\right) \geq M$. In the latter case the problem is infeasible.

In order to solve the tree height reduction problem we generate at most $n$ minimum $s-t$ cuts in $N$. Every minimum $s-t$ cut in network $N$ can be found in $O(n)$ time, since $N-\{t\}$ is an arborescence (see e.g., Hochbaum [15] or Vygen [22]). Also the updating of network $N$ takes at most $O(n)$ time. Hence we get

Theorem 3.3 The tree height reduction problem can be solved in $O\left(n^{2}\right)$ time where $n$ is the number of edges on the given tree.

In the next section the tree height reduction problem will be used as a subproblem for balancing the heights of two rooted trees.

\section{Optimal height balancing of two rooted trees}

Let $T^{\prime}$ and $T^{\prime \prime}$ be two trees rooted in vertices $s^{\prime}$ and $s^{\prime \prime}$, respectively. The problem to balance the height of $T^{\prime}$ and $T^{\prime \prime}$ consists in modifying (increasing or reducing) the edge lengths $\ell(e)$ for all $e \in E\left(T^{\prime}\right), E\left(T^{\prime \prime}\right)$ at minimum toal cost so that the height of $T^{\prime}$ and the height of $T^{\prime \prime}$ are equal with respect to the new edge lengths. Let $c^{+}(e)$ and $c^{-}(e)$ be the cost coefficients for increasing and reducing $\ell(e)$, respectively. Moreover, suppose that $\ell^{+}(e)$ and $\ell^{-}(e)$ are the maximum amounts by which the length $\ell(e)$ is allowed to be 
increased and reduced, respectively. Therefore, in the height balancing of $T^{\prime}$ and $T^{\prime \prime}$ we either increase $\ell(e)$ by an amount $x(e)$ or reduce $\ell(e)$ by a amount $y(e)$ within the given bounds $0 \leq x(e) \leq \ell^{+}(e)$ and $0 \leq y(e) \leq \ell^{-}(e)$ for all $e \in E\left(T^{\prime}\right), E\left(T^{\prime \prime}\right)$ such that the total cost

$$
\sum_{e \in E\left(T^{\prime} \cup T^{\prime \prime}\right)}\left(c^{+}(e) x(e)+c^{-}(e) y(e)\right)
$$

becomes minimum and $h_{\tilde{\ell}}\left(T^{\prime}\right)=h_{\tilde{\ell}}\left(T^{\prime \prime}\right)$ under the new edge lengths $\tilde{\ell}$.

Note that if equality $h_{\ell}\left(T^{\prime}\right)=h_{\ell}\left(T^{\prime \prime}\right)$ holds for the original edge lengths $\ell$, then the two rooted trees $T^{\prime}$ and $T^{\prime \prime}$ are already balanced. Otherwise we have to change the edge lengths on $T^{\prime}$ and $T^{\prime \prime}$. Without loss of generality we may assume that $h_{\ell}\left(T^{\prime}\right) \geq h_{\ell}\left(T^{\prime \prime}\right)$. The solution method is based on the following lemmata.

Lemma 4.1 In order to balance the heights of $T^{\prime}$ and $T^{\prime \prime}$ it is sufficient to reduce the height of $T^{\prime}$ and to increase the height of $T^{\prime \prime}$ at minimum total cost subject to the modification bounds until $T^{\prime}$ and $T^{\prime \prime}$ have equal heights.

Proof. Since the cost for increasing or decreasing an edge are positive, every increase of an edge in $T^{\prime}$ or decrease of an edge in $T^{\prime \prime}$ would cause avoidable cost.

Thus it is sufficient to reduce edge lengths in $T^{\prime}$ and to increase edge lengths in $T^{\prime \prime}$. In particular we get the following lemma:

Lemma 4.2 If $\left(x^{*}, y^{*}\right)$ is an optimal edge length modification needed for the height balancing of $T^{\prime}$ and $T^{\prime \prime}$, then there exists exactly one path $P_{s^{\prime \prime}} z^{0}$ from $s^{\prime \prime}$ to a leaf $z^{0} \in Z\left(T^{\prime \prime}\right)$ that contains all edges $e \in E\left(T^{\prime \prime}\right)$ with $x^{*}(e)>0$.

Proof. Since for every $e \in E\left(T^{\prime \prime}\right)$ the cost coefficients $c^{+}(e)$ are positive, increasing the edge lengths on more than one path $P_{s^{\prime \prime}} z, z \in Z\left(T^{\prime \prime}\right)$, implies additional cost.

From Lemma 4.1 and Lemma 4.2 we conclude that for balancing the heights of $T^{\prime}$ and $T^{\prime \prime}$ it is required to reduce the height of tree $T^{\prime}$ and to find one path $P_{s^{\prime \prime} z^{0}}$ from $s^{\prime \prime}$ to a leaf $z^{0}$ in $T^{\prime \prime}$, the so-called best candidate path, whose length is increased such that the heights of $T^{\prime}$ and $T^{\prime \prime}$ become equal.

The minimum height of tree $T^{\prime}$ can be computed in the following way. Let $Z\left(T^{\prime}\right)$ be the set of leaves of $T^{\prime}$. Every path $P_{s^{\prime} z}$ from $s^{\prime}$ to a leaf $z \in Z\left(T^{\prime}\right)$ can be reduced at most by the amount

$$
\ell^{-}\left(P_{s^{\prime} z}\right)=\sum_{e \in E\left(P_{s^{\prime} z}\right)} \ell^{-}(e)
$$

Therefore, the shortest possible height $h_{\min }$ of $T^{\prime}$ is given by

$$
h_{\min }=\max _{z \in Z\left(T^{\prime}\right)}\left\{\ell\left(P_{s^{\prime} z}\right)-\ell^{-}\left(P_{s^{\prime} z}\right)\right\}
$$

This means that tree $T^{\prime}$ can be reduced by an amount $\delta \leq \delta_{\max }$ where $\delta_{\max }$ is given by

$$
\delta_{\max }=h_{\ell}\left(T^{\prime}\right)-h_{\min } .
$$


On the other hand, Lemma 4.1 implies that it is not necessary to reduce the height of tree $T^{\prime}$ below the height of tree $T^{\prime \prime}$. Thus the maximum meaningful amount for a reduction of the height of tree $T^{\prime}$ is given by

$$
\Delta\left(T^{\prime}, T^{\prime \prime}\right)=\min \left(\delta_{\max }, h_{\ell}\left(T^{\prime}\right)-h_{\ell}\left(T^{\prime \prime}\right)\right) .
$$

For reducing the height of tree $T^{\prime}$ by the amount $\Delta\left(T^{\prime}, T^{\prime \prime}\right)$ we generate $q$ minimum $s-t$ cuts $K_{1}, \ldots, K_{q}$ such that

$$
\Delta\left(T^{\prime}, T^{\prime \prime}\right) \leq \sum_{j=1}^{q} \delta\left(K_{j}\right) .
$$

Recall that for every $j=1, \ldots, q$, the corresponding capacity $c\left(K_{j}\right)$ of the minimum $s-t$ cut $K_{j}$ is the minimum cost for reducing the height of $T^{\prime}$ by the amount $\delta\left(K_{j}\right)$. The problem to equalize the height of $T^{\prime}$ with the length $\ell\left(P_{s^{\prime \prime} z}\right)$ of a path from $s^{\prime \prime}$ to a leaf $z$ in the tree $T^{\prime \prime}$ under minimum cost $C_{z}$ can be formulated as the following linear optimization problem:

$$
\begin{aligned}
\left(\mathrm{LP}_{\mathrm{z}}\right) \quad \min _{\tau, x} \sum_{j=1}^{q} c\left(K_{j}\right) \tau\left(K_{j}\right)+\sum_{e \in E\left(P_{s^{\prime \prime} z}\right)} c^{+}(e) x(e) \\
\text { subject to } \sum_{j=1}^{q} \tau\left(K_{j}\right)+\sum_{e \in E\left(P_{s^{\prime \prime} z}\right)} x(e)=h_{\ell}\left(T^{\prime}\right)-\ell\left(P_{s^{\prime \prime} z}\right), \\
0 \leq \tau\left(K_{j}\right) \leq \delta\left(K_{j}\right) \quad \text { for } j=1, \cdots, q, \\
0 \leq x(e) \leq \ell^{+}(e) \quad \text { for all } e \in E\left(P_{s^{\prime \prime}}\right),
\end{aligned}
$$

where $\tau\left(K_{j}\right)$ is the amount by which all the edges of the cut set $E\left(K_{j}\right)$ are reduced.

Without loss of generality we may assume that $\ell^{+}(e)>0$ for every $e \in E\left(P_{s^{\prime \prime} z}\right)$. If there exists an edge $e$ with $\ell^{+}(e)=0$, then its corresponding variable is removed from problem $\left(\mathrm{LP}_{\mathrm{z}}\right)$. On the other hand we know that the cut values $\delta\left(K_{j}\right), j=1, \cdots, q$, are positive. This allows us to reduce $\left(\mathrm{LP}_{\mathrm{z}}\right)$ to a continuous knapsack problem by introducing the following new variables

$$
\begin{aligned}
\xi^{-}\left(K_{j}\right) & =\frac{\tau\left(K_{j}\right)}{\delta\left(K_{j}\right)} & & \text { for } j=1, \ldots, q, \\
\xi^{+}(e) & =\frac{x(e)}{\ell^{+}(e)} & & \text { for all } e \in E\left(P_{s^{\prime \prime} z}\right), \\
\tilde{c}^{-}\left(K_{j}\right) & =\delta\left(K_{j}\right) c\left(K_{j}\right) & & \text { for } j=1, \ldots, q, \\
\tilde{c}^{+}(e) & =\ell^{+}(e) c^{+}(e) & & \text { for all } e \in E\left(P_{s^{\prime \prime}} z\right),
\end{aligned}
$$

Thus we get 


$$
\begin{aligned}
\left(\mathrm{KP}_{\mathrm{z}}\right) \quad \text { minimize } & \sum_{j=1}^{q} \tilde{c}^{-}\left(K_{j}\right) \xi^{-}\left(K_{j}\right)+\sum_{e \in E\left(P_{s^{\prime \prime}}\right)} \tilde{c}^{+}(e) \xi^{+}(e) \\
\text { subject to } & \sum_{j=1}^{q} \delta\left(K_{j}\right) \xi^{-}\left(K_{j}\right)+\sum_{e \in E\left(P_{s^{\prime \prime} z}\right)} \ell^{+}(e) \xi^{+}(e)=h_{\ell}\left(T^{\prime}\right)-\ell\left(P_{s^{\prime \prime} z}\right), \\
& 0 \leq \xi^{-}\left(K_{j}\right) \leq 1 \quad \text { for } j=1, \cdots, q, \\
& 0 \leq \xi^{+}(e) \leq 1 \quad \text { for all } e \in E\left(P_{s^{\prime \prime} z}\right) .
\end{aligned}
$$

If the inequality

$$
\sum_{j=1}^{q} \delta\left(K_{j}\right)+\sum_{e \in E\left(P_{s^{\prime \prime} z}\right)} \ell^{+}(e) \geq h_{\ell}\left(T^{\prime}\right)-\ell\left(P_{s^{\prime \prime} z}\right)
$$

is satisfied, then problem $\left(\mathrm{KP}_{\mathrm{z}}\right)$ is feasible. Its optimal objective function value $C_{z}$ can be obtained in linear time time by using the solution algorithm of Balas and Zemel, see e.g., [1], [17], [19]. Through the transformations (12) and (13) an optimal solution $(x, \tau)$ of problem $\left(\mathrm{LP}_{\mathrm{z}}\right)$ with the same objective value $C_{z}$ is obtained. Otherwise, if inequality (14) does not hold, then problem $\left(\mathrm{KP}_{\mathrm{z}}\right)$ has no solution. In this case we set $C_{z}=+\infty$. The best candidate path $P_{s^{\prime \prime}} z^{0}$ is obtained by

$$
z^{0} \in \operatorname{argmin}\left\{C_{z}: z \in Z\left(T^{\prime \prime}\right)\right\}
$$

If $C_{z^{0}}=+\infty$, then the given problem is infeasible. Otherwise, we derive an optimal solution of the height balancing problem on trees $T^{\prime}$ and $T^{\prime \prime}$ from the optimal solution $\left(\tilde{\xi}^{+}, \tilde{\xi}^{-}\right)$of the continuous knapsack problem $\left(\mathrm{KP}_{\mathrm{z}^{0}}\right)$ as follows: For $j=1, \ldots, q$ let

$$
y_{j}(e)= \begin{cases}\tilde{\xi}^{-}\left(K_{j}\right) \delta\left(K_{j}\right) & \text { if } e \in E\left(K_{j}\right) \cap E\left(T^{\prime} \cup T^{\prime \prime}\right), \\ 0 & \text { otherwise. }\end{cases}
$$

Now define

$$
y^{*}(e)=\sum_{j=1}^{q} y_{j}(e) \quad \text { for all } e \in E\left(T^{\prime} \cup T^{\prime \prime}\right),
$$

and

$$
x^{*}(e)= \begin{cases}\tilde{\xi}^{+}(e) \ell^{+}(e) & \text { if } e \in E\left(P_{s^{\prime \prime}} z^{0}\right), \\ 0 & \text { otherwise. }\end{cases}
$$

Then $\left(x^{*}, y^{*}\right)$ is an optimal solution vector of the height balancing problem on the trees $T^{\prime}$ and $T^{\prime \prime}$.

The solution algorithm has the following complexity: Let $n=\left|E\left(T^{\prime}\right)\right|+\left|E\left(T^{\prime \prime}\right)\right|$. The reduction argument $\delta_{\max }$ is computed in at most $O\left(\left|E\left(T^{\prime}\right)\right|\right)$ time, if we traverse the tree network $T^{\prime}$ in a depth-first manner. Furthermore, according to Theorem 3.3, the reduction of height $h_{\ell}\left(T^{\prime}\right)$ is performed in $O\left(\left|E\left(T^{\prime}\right)\right|^{2}\right)$ time. On the other hand, during the execution of the solution algorithm we solve at most $\left|E\left(T^{\prime \prime}\right)\right|$ continuous knapsack 
problems $\left(\mathrm{KP}_{\mathrm{z}}\right), z \in Z\left(T^{\prime \prime}\right)$. A continuous knapsack problem can be solved in $O\left(\left|E\left(T^{\prime \prime}\right)\right|\right)$ time. Moreover, an optimal solution vector $\left(x^{*}, y^{*}\right)$ of the height balancing problem is obtained from the optimal solution of $\left(\mathrm{KP}_{\mathrm{z}^{0}}\right)$ in $O\left(n^{2}\right)$ time. Thus we conclude that the time complexity of the algorithm is bounded by $O\left(n^{2}\right)$.

Altogether we get

Theorem 4.3 The height of two rooted trees $T^{\prime}$ and $T^{\prime \prime}$ can be balanced under minimum cost in $O\left(n^{2}\right)$ time where $n$ is the total number of edges in $T^{\prime}$ and $T^{\prime \prime}$.

In the next sections we use the balancing of two rooted trees as a subproblem for solving the inverse absolute (and vertex) 1-center location problems.

\section{Inverse absolute 1-center location problem}

\subsection{Inverse absolute 1-center location problem without essential topol- ogy change}

In the following we propose a combinatorial algorithm for the inverse absolute 1-center location problem on the tree network $T$ in which we assume that the given bounds for reducing the edge lengths obey the condition

$$
\exists e \in E\left(P_{s s_{1}}\right) \quad \text { such that } \ell^{-}(e)<\ell(e),
$$

where $P_{s s_{1}}$ is the unique path from the prespecified vertex $s$ to the nearest vertex $s_{1} \in$ $E(L)$ with $\operatorname{deg}\left(s_{1}\right) \geq 3$ as introduced in Subsection 2.2. This condition implies that an essential topology change never occurs on the tree $T$. We want to modify the edge lengths of $T$ at minimum total cost within given bounds such that $s$ becomes the absolute 1-center under the new edge lengths.

Let $\operatorname{deg}(s) \geq 2$ and consider the subtrees $L$ and $R$ of $T$ as explained in Subsection 2.2. We root $L$ and $R$ in vertex $s$. Now, if

$$
h_{\ell}(L)=h_{\ell}(R),
$$

then there exists a path $P_{s z^{*}}$ in tree $L$ with $\ell\left(P_{s z^{*}}\right)=h_{\ell}(L)$ and a path $P_{s z^{0}}$ in tree $R$ with $\ell\left(P_{s z^{0}}\right)=h_{\ell}(R)$. Thus, according to the definition of the height of a tree, the path $P_{z^{*} z^{0}}$ is a longest path of $T$ containing $s$ as midpoint. This implies that the prespecified vertex $s$ is the wanted absolute 1-center. Otherwise we have to change the edge lengths in $T$. The following lemma is basic for the solution method.

Lemma 5.1 In order to solve the inverse absolute 1-center location problem without essential topology change on the given tree network $T$, it is sufficient to balance the heights of the subtrees $L$ and $R$ both rooted in the prespecified vertex $s$.

Proof. The lemma is an immediate consequence of Theorem 2.1 and the fact that the topology of the tree $T$ does not change by virtue of condition (15). 
Based on all considerations above we can solve the problem under investigation in the following way:

If $\operatorname{deg}(s)=1$, then $s$ can never becomes the midpoint of a longest path and in this case the problem is infeasible. Otherwise, in the first step we partition tree $T$ into subtrees $L$ and $R$ according to (4) and root both of these subtrees in vertex $s$. Then we balance the heights of subtrees $L$ and $R$. An optimal solution of the height balancing problem yields the optimal solution of the inverse absolute 1-center location problem under investigation.

Observe that the longest paths from $s$ to the leaves $z \in Z(T)$ are found in $O(n)$ time. Thus the partition of $T$ into subtrees $L$ and $R$ takes $O(n)$ time. Based on Theorem 4.3 the heights of subtrees $L$ and $R$ can be balanced in $O\left(n^{2}\right)$ time. Thus we get

Theorem 5.2 The inverse absolute 1-center location problem with edge length modification can be solved in $O\left(n^{2}\right)$ time on a tree network (with $n$ edges), provided that no essential topology change occurs in the given tree.

\subsection{The general inverse absolute 1-center location problem}

In this subsection, we drop condition (15), i.e., an essential topology change may occur in the tree $T$ during the height reduction of the left tree $L$. This means, after a length reduction in the left tree $L$ some vertices of $L$ may move to the right tree $R$, see Figure 1 and Figure 2. In any reduction step the length of the unique edge $e_{s}=s s_{1}$ in the left tree $L$ which is incident with $s$ is reduced. If this edge length is reduced to 0 and $\operatorname{deg}\left(s_{1}\right)>2$ we have a choice in defining a new left tree $L^{1}$. In principle, $L^{1}$ can be any subtree of $L$ rooted in $s=s_{1}$ with maximum height. The right tree $R^{1}$ is defined as the complement of $L^{1}$ in $T$. Now the height of the new trees $L^{1}$ and $R^{1}$ is balanced. In order to minimize the number of height balancing steps we introduce the notion of the compressed depth $r$ of $T$. Thus the complexity of the final algorithm becomes $O\left(n^{2} r\right)$ with $1 \leq r \leq n$.

Let

$$
\mathcal{Z}=\left\{z \in Z(T): d_{\ell}(s, z)=h_{\ell}(T)\right\} .
$$

Consider the value $\Delta(L, R)$ defined by (11). On every path $P_{s z}$ let $s_{1}(\neq s), s_{2}, \ldots, s_{i(z)}$ be the sequence of vertices from $s$ to $z$ with the following three properties

$$
\begin{gathered}
\operatorname{deg}\left(s_{k}\right) \geq 3, \\
\ell\left(P_{s s_{k}}\right)=\ell^{-}\left(P_{s s_{k}}\right),
\end{gathered}
$$

and

$$
\ell\left(P_{s s_{k}}\right) \leq \Delta(L, R)
$$

for all $k=1,2, \ldots, i(z)$.

Definition 5.3 The compressed depth of $T$ is given by

$$
r=\min \{i(z): z \in \mathcal{Z}\}+1 .
$$

Moreover, a path $P_{s z}, z \in \mathcal{Z}$, with $i(z)=r-1$ is called compressed longest path in $T$. 
Observe that if $r=1$, then the left tree $L$ is a path or at least one of the properties $\ell\left(P_{s s_{1}}\right)=\ell^{-}\left(P_{s s_{1}}\right)$ and $\ell\left(P_{s s_{1}}\right) \leq \Delta(L, R)$ does not hold, where $s_{1}$ is the nearest vertex to $s$ with $\operatorname{deg}\left(s_{1}\right) \geq 3$ on the left tree $L$ as introduced in Section 2. In this case an optimal solution of the problem can be found without an essential topology change on the underlying tree $T$. Hence, the solution algorithm of Subsection 5.1 can be applied. Otherwise we consider a compressed longest path $P_{s z^{*}}$ of $T$. Let $s_{1}, \ldots, s_{r-1}$ be the sequence of its vertices from $s$ to $s_{r-1}$ with properties (16), (17) and (18). Moreover, let $s_{0}=s$.

For any $k, 0 \leq k<r$, let $T^{k}$ be the tree obtained from $T$ by reducing the length $\ell\left(P_{s s_{k}}\right)$ to zero. Moreover, let $L^{k}$ and $R^{k}$ be the two subtrees of $T^{k}$ which are rooted in $s=s_{i}, i=0, \ldots, k$, and are determined by (3) or (4). For example, Figure 4 illustrates the resulting tree $T^{2}$ with the two corresponding subtrees $L^{2}$ and $R^{2}$ which is obtained from the tree $T$ in Figure 1 by reducing the length of the path $P_{s s_{2}}$ to zero.

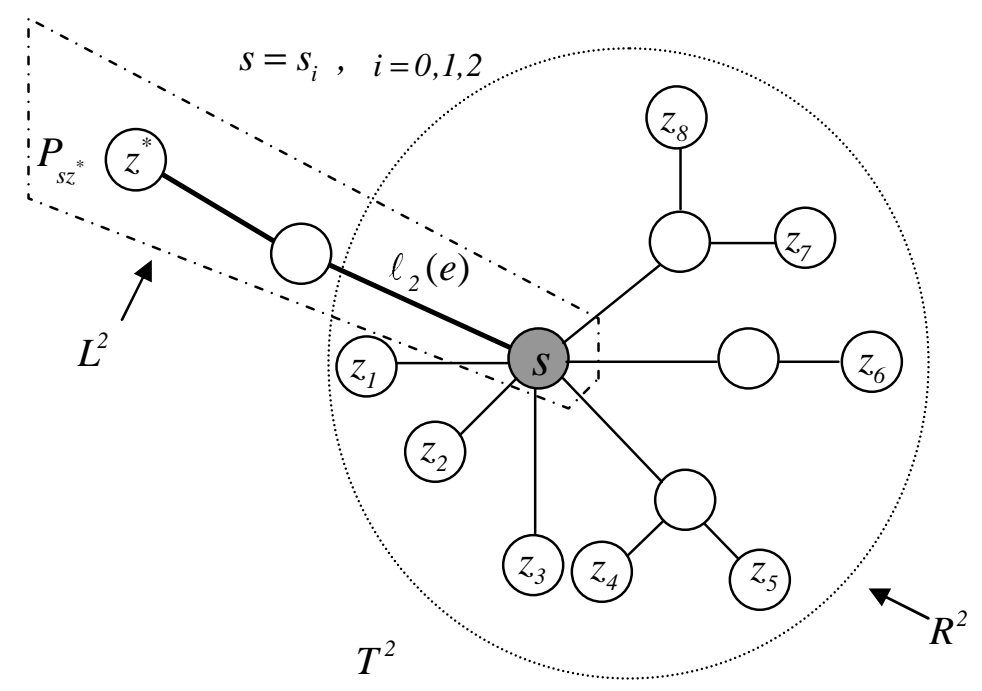

Figure 4: $T^{2}$ is obtained from the tree $T$ of Figure 1 by reducing the length of $P_{s s_{2}}$ to zero. $P_{s z^{*}}$ is a compressed longest path of $T$.

Subproblem $\left(S P_{k}\right)$ consists in finding the minimum cost $C\left(T^{k}\right)$ incurred by changing the edge lengths of tree $T^{k}$ such that $s$ becomes an absolute 1-center of tree $T^{k}$. Thus the total cost in Step $k$ is given by

$$
C_{k}=C_{s, k}+C\left(T^{k}\right)
$$

where

$$
C_{s, k}=\sum_{e \in E\left(P_{s s_{k}}\right)} c^{-}(e) \ell(e)
$$

is the required cost for reducing $\ell\left(P_{s s_{k}}\right)$ to 0 . The minimum $\operatorname{cost} C_{k_{0}}$ with

$$
k_{0} \in \operatorname{argmin}\left\{C_{k}: k=0, \ldots, r-1\right\}
$$


yields the optimum objective function value of the general inverse absolute 1-center location problem on tree $T$. In the case that $C_{k_{0}}$ has a finite value, an optimal solution of the problem is obtained by

$$
\begin{aligned}
& y^{*}(e)= \begin{cases}\ell(e) & \text { if } e \in E\left(P_{s s_{k_{0}}}\right), \\
y_{k_{0}}(e) & \text { otherwise, }\end{cases} \\
& x^{*}(e)= \begin{cases}0 & \text { if } e \in E\left(P_{s s_{k_{0}}}\right), \\
x_{k_{0}}(e) & \text { otherwise. }\end{cases}
\end{aligned}
$$

where $\left(x_{k_{0}}, y_{k_{0}}\right)$ is the optimal solution of the height balancing problem applied to tree $T^{k_{0}}$.

Note that the tree $T^{k}$ is obtained from $T^{k-1}$ by reducing the length $\ell\left(P_{s_{k-1} s_{k}}\right)$ to 0 and the cost $C_{s, k}$ can be also computed in a recursive way by

$$
C_{s, k}=C_{s, k-1}+\sum_{e \in E\left(P_{s_{k-1} s_{k}}\right)} c^{-}(e) \ell(e) .
$$

Moreover, we consider the following two conditions during the computation of $\operatorname{cost} C_{i}$, $i=0, \ldots, k$ :

Condition 1. If

$$
\sum_{e \in E\left(P_{s_{k-1} s_{k}}\right)} c^{-}(e) \ell(e) \geq \min _{0 \leq i \leq k-1} C\left(T^{i}\right),
$$

we can skip the computation of $C_{i}, i \geq k$, since none Subproblem $\left(S P_{i}\right)$ can improve the best objective function value already found.

Condition 2. Observe that after any optimal modification the path $P_{s z^{*}}$ remains a longest path from $s$ to the leaves. Therefore, if for a given $k \in\{1, \ldots, r-1\}$ inequality

$$
\ell\left(P_{s_{k-1} s_{k}}\right)>h_{\ell}\left(L^{k-1}\right)-h_{\ell}\left(R^{k-1}\right)
$$

holds with respect to the edge lengths of $T^{k-1}$, then an optimal solution never reduces $\ell\left(P_{s_{k-1} s_{k}}\right)$ to 0 . In this case we may also skip the computation of $C_{i}, i \geq k$.

If for some $k \in\{1, \ldots, r-1\}$ at least one of the inequalities (22) and (23) holds, then we set $C_{i}=+\infty$ for all $i=k, \ldots, r-1$.

Based on the previous considerations the solution algorithm for the general inverse absolute 1-center location problem on a tree network $T$ can be summarized as follows:

1. Find the compressed depth $r$ and a compressed longest path $P_{s z^{*}}, z^{*} \in Z(T)$.

2. Solve Subproblem $\left(S P_{0}\right)$. If $r=1$ or $C_{0}=0$, then the original problem has been solved. Otherwise, determine index $k_{0}$ according to (19) under consideration of Conditions 1 and 2. If the corresponding $\operatorname{cost} C_{k_{0}}$ is finite, then an optimal solution vector $\left(x^{*}, y^{*}\right)$ of the original problem is given by (20) and (21), else we conclude that the problem is infeasible. 
Now we are going to compute the running time of this solution approach. A compressed longest path of $T$ is found in $O(n)$ time if $T$ is traversed in a depth-first search manner. The total time needed to construct the trees $T^{k}, k=1, \ldots, r-1$, is $O(n)$. Moreover, due to Theorem 5.2 we know that the solution algorithm of Subsection 5.1 runs in $O\left(n^{2}\right)$ time. On the other hand, during execution of this method we recall the solution algorithm of Subsection 5.1 at most $r$ times where $r<\frac{n}{2}+1$. Therefore the overall time complexity of the solution algorithm for the general model is bounded by $O\left(n^{2} r\right)$. Observe that $r \leq \log (n)$ when the underlying tree $T$ is a complete binary tree with root $s$. Thus we get

Theorem 5.4 The general inverse absolute 1-center location problem with edge length modifications can be solved in $O\left(n^{2} r\right)$ time on a tree network where $r$ is the compressed depth of the given tree and $n$ is the number of the edges of $T$.

\section{Inverse vertex 1-center location problem}

This section considers the inverse vertex 1-center location problem on the given tree network T. Recently Yang and Zhang [23] stated a solution method which solves the problem in $O\left(n^{2} \log n\right)$ time provided that the modified edge lengths always remain positive, i.e., $\tilde{\ell}>0$. Moreover, they pointed out that the general problem, i.e., in case of $\tilde{\ell} \geq 0$, can be solved in $O\left(n^{3} \log n\right)$. However, a solution procedure was not discussed in this case. In the following we derive new solution methods with improved time complexities for the inverse vertex 1-center problem using the tree height balancing algorithm.

\subsection{Inverse vertex 1-center location problem with positive modified edge lengths}

In this subsection we investigate the inverse vertex 1-center location problem in which we assume that

$$
\ell^{-}(e)<\ell(e) \text { for all } e \in E(T) .
$$

This condition guarantees that during solving the problem the modified edge lengths always remain positive, i.e., $\tilde{\ell}>0$. Let $\hat{L}$ and $\hat{R}$ be two subtrees of $T$ which are determined according to (5) and (6) and are rooted in the vertices $a(s)$ and $s$, respectively. Lemma 2.3 yields immediately the following result

Lemma 6.1 Under condition (24) it is sufficient to balance the heights of the subtrees $\hat{L}$ and $\hat{R}$ for solving the inverse vertex 1-center location problem on a given tree $T$.

Note that the subtrees $\hat{L}$ and $\hat{R}$ can be determined in $O(n)$ time if we traverse the tree $T$ in a depth-first search manner. On the other hand, based on Theorem 4.3 the balancing the heights of $\hat{L}$ and $\hat{R}$ is performed in $O\left(n^{2}\right)$ time. Thus we get

Theorem 6.2 The inverse vertex 1-center location problem with positive modified edge lengths can be solved in $O\left(n^{2}\right)$ time on a tree with $n$ edges. 


\subsection{The general inverse vertex 1-center location problem}

In the following we describe a solution method with improved time complexity for the general inverse vertex 1-center location problem on the given tree network $T$ dropping condition (24).

We start with defining the minimal repeat number $r_{v}$ on tree $T$. On every path $P_{s z}$, $z \in Z(T)$, let $s_{1}, s_{2}, \ldots, s_{j(z)}$ with $s \neq s_{1}$ denote the sequence of all vertices from $s$ to the leaf $z$ which satisfy the properties (17) and (18) for all $k=1,2, \ldots, j(z)$. Recall that

$$
\mathcal{Z}=\left\{z \in Z(T): d_{\ell}(s, z)=h_{\ell}(T)\right\} .
$$

Definition 6.3 Given a tree $T$, the minimal repeat number $r_{v}$ is defined as

$$
r_{v}=\min \{j(z): z \in \mathcal{Z}\}+1 .
$$

Obviously, $r_{v} \leq n$. If $r=1$, then an optimal solution of the problem can be found as in Subsection 6.1. Otherwise we consider a path $P_{s z^{*}}$ of $T$ with $j\left(z^{*}\right)=r_{v}-1$. Let $s_{1}, s_{2}, \ldots, s_{r_{v}-1}$ be the sequence of its vertices from $s$ to $z^{*}$ with properties (17) and (18). Further, we set $s_{0}=s$. Recall that $T^{k}$ is the tree obtained from $T$ by reducing the length $\ell\left(P_{s s_{k}}\right), 0 \leq k<r_{v}$, to 0 . Moreover, let $\hat{L}^{k}$ and $\hat{R}^{k}$ be two subtrees of $T^{k}$ which are determined according to (5)-(6) and are rooted in $a\left(s_{k}\right)$ and $s=s_{i}, i=0, \ldots, k$, respectively.

For every tree $T^{k}$ we determine a corresponding total cost $C_{k}$ according to

$$
C_{k}=C_{s, k}+\hat{C}\left(T^{k}\right)
$$

where the cost $C_{s, k}$ is incurred by reducing the length $\ell\left(P_{s s_{k}}\right)$ to 0 and $\hat{C}\left(T^{k}\right)$ is the optimal value obtained by balancing the height of the subtrees $\hat{L}^{k}$ and $\hat{R}^{k}$ of $T^{k}$. If

$$
\sum_{e \in E\left(P_{s_{k-1} s_{k}}\right)} c^{-}(e) \ell(e) \geq \min _{0 \leq i \leq k-1} \hat{C}\left(T^{i}\right),
$$

or

$$
h_{\ell}\left(\hat{L}^{k-1}\right) \leq h_{\ell}\left(\hat{R}^{k-1}\right),
$$

hold, then we can skip the computation of $C_{i}, i \geq k$, and assign immediately $C_{i}=+\infty$ for all $i \geq k$.

By choosing an index $k_{0}$ with

$$
k_{0} \in \operatorname{argmin}\left\{C_{k}: k=0, \ldots, r_{v}-1\right\},
$$

we obtain an optimal solution of the general inverse vertex 1-center location problem on the given tree network $T$ with cost $C_{k_{0}}$ by (20) and (21).

Since for solving the general inverse vertex 1-center location problem at most $r_{v}$ height balancing problems have to be solved we get

Theorem 6.4 The general inverse vertex 1-center location problem with edge length modification can be solved in $O\left(n^{2} r_{v}\right)$ time on a tree network where $r_{v}$ is the minimal repeat number of the given tree with $n$ edges. 


\section{Concluding remarks}

In this article we investigated the inverse absolute and the inverse vertex 1-center location problems with variable edge lengths on tree networks. It was shown that the problems can be formulated as nonlinear semi-infinite (or nonlinear) programming problems. Exact combinatorial solution algorithms with fast running times were developed.

A comparison of the solution approach of Yang and Zhang [23] for the inverse vertex center problem and the method described in this paper shows the following. In both methods the given tree is split into two subtrees whose height has to be balanced. The main difference lies in the height balancing procedure. Yang and Zhang balance the height by solving a min cost network flow problem in a 2-terminal series parallel graph and they apply linear programming duality to evaluate the solution. This paper, however, solves first a series of min cut problems in planar networks in a preprocessing step. The cut values obtained from these problems are used in a continuous knapsack problem (solvable in linear time) for the height balancing problem.

In case that the underlying tree network is a star graph, the inverse absolute and inverse vertex 1-center location problems can be solved efficiently in $O(n)$ time by straightforward approaches.

\section{Acknowledgments}

The authors thank Elisabeth Gassner and Ulrich Pferschy for their constructive suggestions during the preparation of this paper. Moreover, they are grateful to anonymous referees for their helpful comments.

\section{References}

[1] E. Balas, E. Zemel, An algorithm for large zero-one knapsack problems, Operations Research 28(1980), 1130-1154.

[2] R.E. Burkard, M. Galavii and E. Gassner, The inverse Fermat-Weber problem, Technical Report 2008-14, Graz University of Technology, Graz, 2008.

[3] R.E. Burkard, C. Pleschiutschnig and J. Zhang, Inverse median problems, Discrete Optimization 1(2004), 23-39.

[4] R.E. Burkard, C. Pleschiutschnig and J. Zhang, The inverse 1-median problem on a cycle, Discrete Optimization 5(2007), 242-253.

[5] M.C. Cai, X.G. Yang and J.Z. Zhang, The complexity analysis of the inverese center location problem, Journal of Global Optimization 15(1999), 213-218.

[6] M.S. Daskin, Network and Discrete Location: Modeles, Algorithms and Applications, John Wiley, New York, 1995.

[7] Z. Drezner and H.W. Hamacher, Facility Location, Applications and Theory, Springer Verlag, Berlin, 2004.

[8] R.L. Francis, L.F. McGinnis and J.A. White, Facility Layout and Location, An Analytical Approach, Prentice Hall, Englewood Cliffs, 1992. 
[9] M. Galavii, Inverse 1-Median Problems, Ph.D. Thesis, Institute of Optimization and Discrete Mathematics, Graz University of Technology, Graz, 2008.

[10] E. Gassner, The inverse 1-maxian problem with edge length modification, J. Combinatorial Optimization 16(2007), 50-67.

[11] E. Gassner, An inverse approach to convex ordered median problems in trees, Technical Report 2008-16, Graz University of Technology, Graz, 2008.

[12] G.Y. Handler, Minimax location of a facility in an undirected tree graph, Transportation Science 7(1973), 287-293.

[13] J. Hatzl, personal communication, 2006.

[14] C. Heuberger, Inverse combinatorial optimization: A survey on problems, methods, and results, Journal of Combinatorial Optimization 8(2004), 329-361.

[15] D.S. Hochbaum, The pseudoflow algorithm: A new algorithm for the maximum flow problem, Operations Research, to appear.

[16] O. Kariv and S. L. Hakimi, An algorithmic approach to network location problems. I: The p-centers, SIAM J. Appl. Math. 37(1979), 513-538.

[17] H. Kellerer, U. Pferschy and D. Pisinger, Knapsack Problems, Springer Verlag, Berlin, 2004.

[18] R.F. Love, J.G. Morris and G.O. Wesolowsky, Facilities Location: Models and Methods, North-Holland, New York, 1988.

[19] S. Martello and P. Toth, Knapsack Problems: Algorithms and Computer Implementations, John Wiley, Chichester, 1990.

[20] N. Megiddo, Linear-time algorithms for linear programming in $\mathbb{R}^{3}$ and related problems, SIAM J. Comput. 6(2007), 5-16.

[21] B.P. Mirchandani, R.L. Francis, Discrete Location Theory, John Wiley, New York, 1990.

[22] J. Vygen, On dual minimum cost flow algorithms, Mathematical Methods of Operations Research 56(2002), 101-126.

[23] X. Yang and J. Zhang, Inverse center location problem on a tree, Journal of Systems Science and Complexity 21(2008), 651-664.

[24] J. Zhang, Z. Liu and Z. Ma, Some reverse location problems, European Journal of Operational Research 124(2000), 77-88.

\section{Appendix}

The following notations are used in the paper:

- $G=(V(G), E(G))$ : a graph with vertex set $V(G)$ and edge set $E(G)$

- $T=(V(T), E(T))$ : a tree network with vertex set $V(T),|V(T)|=n+1$ and edge set $E(T)$ 
- $\ell(e)$ : length of edge $e$

- $d_{\ell}(u, v)$ : shortest path distance between two points $u$ and $v$ of $G$ with respect to edge lengths $\ell$

- $\ell^{+}(e), \ell^{-}(e)$ : maximum amounts by which $\ell(e)$ can be increased or reduced, respectively

- $c^{+}(e), c^{-}(e)$ : cost coefficients for increasing and reducing $\ell(e)$, respectively

- $x(e), y(e)$ : amounts of increasing and reducing $\ell(e)$, respectively

- $P_{u v}$ : unique path in $T$ from vertex $u$ to $v$

- $\ell\left(P_{u v}\right)$ : length of path $P_{u v}$

- $h_{\ell}(T)$ : height of a rooted tree $T$ with respect to edge lengths $\ell$

- $Z(T)$ : set of leaves of tree $T$

- $z$ : a leaf of the tree

- $s$ : a predetermined vertex of the given tree (the prospective center)

- $P_{s z^{*}}$ : a longest path from $s$ to the leaves of $T$

- $a(s)$ : adjacent vertex to $s$ on the longest path $P_{s z^{*}}$

- $\operatorname{deg}(v)$ : degree of vertex $v$

- $r$ : compressed depth of tree $T$

- $r_{v}$ : minimal repeat number with respect to tree $T$ 